\title{
Upregulation of Multidrug Resistance-Associated Protein 1 by Allyl Isothiocyanate in Human Bronchial Epithelial Cell: Involvement of c-Jun N-Terminal Kinase Signaling Pathway
}

\author{
Shujun Wang, ${ }^{1}$ Shanshan Wang, ${ }^{1,2}$ Chenyin Wang, ${ }^{1,3}$ Yajun Chen, ${ }^{1}$ Jie Li, ${ }^{1}$ Xueqi Wang, \\ Dianlei Wang, ${ }^{1}$ Zegeng Li, ${ }^{4}$ Zhaoliang Peng, ${ }^{1}$ and Ling Fan ${ }^{1}$ \\ ${ }^{1}$ Anhui University of Chinese Medicine, Hefei, Anhui 230038, China \\ ${ }^{2}$ People's Hospital of Feidong County, Hefei, Anhui 230001, China \\ ${ }^{3}$ Faculty of Health Sciences, University of Macau, Macau \\ ${ }^{4}$ The First Affiliated Hospital to Anhui University of Chinese Medicine, Hefei, Anhui 230031, China
}

Correspondence should be addressed to Dianlei Wang; dlwang@ahtcm.edu.cn and Zegeng Li; 395693237@qq.com

Received 13 May 2015; Accepted 24 June 2015

Academic Editor: Silvana Hrelia

Copyright ( 2015 Shujun Wang et al. This is an open access article distributed under the Creative Commons Attribution License, which permits unrestricted use, distribution, and reproduction in any medium, provided the original work is properly cited.

\begin{abstract}
Multidrug resistance-associated protein 1 (MRP1) plays a protective role in the etiology and progression of chronic obstructive pulmonary disease (COPD) which results from oxidative stress and inflammation of lung injury. The lower functional MRP1 activity is related to COPD development. Our previous study showed that Allyl isothiocyanate (AITC) induced the expression and activity of MRP1 in a dose-dependent manner. However, which signaling pathway contributes to the upregulation of MRP1 by AITC is unclear. In this study, signaling pathway specific inhibitors were used to examine the mechanism of AITC. We found that JNK inhibitor SP600125 treatment decreased MRP1 mRNA expression in 16HBE14o- cells. But the ERK inhibitor U0126 or PI3K/Akt inhibitor LY294002 produced no obvious effect. The AITC-induced increase of MRP1 mRNA expression was abolished by cotreatment of SP600125, while it was not obviously affected by U0126 or LY294002. Furthermore, AITC acivates the JNK signaling pathway in 16HBE14o- cells. Finally, we found that JNK pathway mediated the upregulation of AITC-induced expression and function of MRP1. Taken together, our results indicated that AITC increased the expression and the activity of MRP1 via a JNK-dependent pathway. ERK and PI3K signaling pathway were not involved in the expression of MRP1 mRNA.
\end{abstract}

\section{Introduction}

Both smoking and ambient airborne particulate matter (PM) with an aerodynamic diameter less than $2.5 \mu \mathrm{m}$ (PM2.5) are capable of inducing noxious particles or gases in the lung [1], which are the principal risk factors for chronic obstructive pulmonary disease (COPD). Proteins of the ATP-binding cassette $(\mathrm{ABC})$ superfamily, such as the multidrug resistanceassociated protein 1 (MRP1), play an important role in normal physiology by protecting tissues from toxic xenobiotics and endogenous metabolites $[2,3]$. In normal lung tissue, the protein level of MRP1 is high in bronchial epithelium. However, bronchial biopsies of COPD patients indicated that the protein expression of MRP1 was lower in patients with COPD than healthy humans [4]. In our previous study, the results showed that the protein expression of the bronchial epithelial MRP1 was significantly decreased in papain and smoking induced COPD rat model [5]. So far, MRP1 is known as an essential factor for maintaining tissue homeostasis to defend certain tissues against the stress from xenobiotic insults, proinflammatory cysteinyl, and a vast array of other endoand xenobiotic organic anions [6].

Several signaling pathways, such as mitogen activated protein kinase (MAPK) and phosphatidylinositide 3-kinase/ serine-threonine kinase (PI3K/Akt), were reported to regulate the expression of MRP1 [7-9]. Earlier studies indicated that the classical MAPK pathways play an important role in regulating gene expression in eukaryotic cells and they link extracellular signals to the machinery that controls fundamental cellular processes $[10,11]$. Among these pathways, 
c-Jun NH2-terminal kinase (JNK) and extracellular signalregulated kinase (ERK) were found to be involved in MRP1mediated multidrug resistance of malignant tumor cells [7, 12]. In addition, PI3K/Akt signaling pathway is another important intracellular signaling pathway which is also involved in the drug resistance of different types of human neoplasm cells $[13,14]$. LY294002, a PI3K-specific inhibitor, has been reported to reduce the activity of the MRP1 promoter by stimulating vascular endothelial growth factor (VEGF) [15]. In addition, treatment of K562 cell line with LY294002 or Akt siRNA downregulated P-glycoprotein (Pgp) and MRP1 expression [9]. Although these studies are informative and suggesting a possible relationship between these signaling pathways and MRP1 expression, the mechanism between these three pathways and MRP1 expression in human bronchial epithelial cell line needs to be further studied.

The compounds isothiocyanates (ITCs) from many cruciferous vegetables like cabbage and cauliflower were reported to be useful for treating COPD. Many ITCs have been demonstrated to have chemopreventive activity in a number of inflammatory disorders and modulation of cellular redox status [16-18]. Up to now, most studies were focused on the effect that isothiocyanates can reverse multidrug resistance mediated by MRP1 in cancer cells $[19,20]$. However, little is known regarding the effect of isothiocyanates on the expression of MRP1 in normal lung tissue. Allyl isothiocyanate (AITC), a structurally related compound of isothiocyanates, can improve the lung function of COPD rats which is induced by cigarette smoke [21]. Our previous study found that AITC can increase the expression and activity of MRP1 in 16HBE14o- cells [22]. However, the mechanisms remain unknown. Therefore, in this study, we tried to elucidate the contribution of the ERK1/2, JNK, and PI3K/Akt signaling pathways to AITC upregulation of MRP1 activity and expression in 16HBE14o- cells.

\section{Materials and Methods}

2.1. Reagents. AITC was purchased from Anhui Haibei Import and Export Company (Hefei, Anhui, China). RPMI 1640 medium and Fetal Bovine Serum were purchased from Gibco. LY294002, U0126, SP600125, 5-CFDA, and sodium dodecyl sulfate (SDS) and dimethyl sulphoxide (DMSO) were purchased from Sigma-Aldrich (Oakville, ON, Canada). Monoclonal antibodies, including anti-MRP1, $\beta$-actin, and JNK, were purchased from Santa Cruz Biotechnology (Santa Cruz, CA, USA). p-JNK antibody was purchased from Cell Signaling Technology (Danvers, MA, USA). Trizol reagent was purchased from Invitrogen (Carlsbad, CA, USA).

2.2. Cell Culture and Treatments. The human bronchial epithelial cell line 16HBE14o- was purchased from Shanghai Fuxiang Biological Technology Company (Shanghai, China). Cells were grown in RPMI-1640 medium supplemented with $10 \%$ FBS. Cells were maintained at $37^{\circ} \mathrm{C}$ in $5 \% \mathrm{CO}_{2}$ atmosphere. Cells were routinely subcultured prior to reaching $80 \%$ confluence. AITC was dissolved in DMSO. The previous study showed that AITC $(40 \mu \mathrm{M})$ had no significant effect on cell viability of $16 \mathrm{HBE} 14 \mathrm{o}-$ after incubating for $24 \mathrm{~h}$ [22]. Therefore, the 16HBE14o- cells were cultured in serum-free medium with AITC $(40 \mu \mathrm{M})$ for $24 \mathrm{~h}$. In the meantime, control cells were treated with $0.1 \%$ DMSO only. To explore possible signaling pathways, $16 \mathrm{HBE} 14 \mathrm{o}-$ cells were preincubated with either ERK kinase inhibitor U0126 $(20 \mu \mathrm{M})$ or JNK inhibitor SP600125 $(20 \mu \mathrm{M})$ or PI3K/Akt inhibitor LY294002 $(10 \mu \mathrm{M})$ for $30 \mathrm{~min}$ and $60 \mathrm{~min}$ before the addition of AITC.

2.3. RNA Extraction and Real-Time Polymerase Chain Reaction (RT-PCR). Total RNA was isolated from 16HBE14ocells, by using TRIzol reagent (Invitrogen, Carlsbad, CA), following the manufacturer's instructions. The concentrations and A260/A280 ratios of the isolated RNAs were determined from the absorbance at 260 and $280 \mathrm{~nm}$ by a Hitachi spectrophotometer (modelU1100) and the integrity was verified by agarose gel electrophoresis. cDNA was generated using the High-Capacity cDNA Archive Kit according to the manufacturer's instructions. Real-time PCR was performed using the SYBR Green Master Mix system (Applied Biosystems, CA, USA) according to the manufacturer's instructions on an ABI 7500 real-time PCR machine (Applied Biosystems). Following the reverse transcription reaction, $2 \mu \mathrm{L}$ of the resultant cDNA was used for PCR amplification reaction as follows: $10 \mathrm{~min}$ at $95^{\circ} \mathrm{C}$, followed by 40 cycles of $15 \mathrm{~s}$ at $95^{\circ} \mathrm{C}$ and $1 \mathrm{~min}$ at $60^{\circ} \mathrm{C}$. Primers pairs for each transcript were MRP1 forward $5^{\prime}$-CCTGGAGCTGGCCCACCTGA-3' and reverse $5^{\prime}$-CGCTGCCCGACACTGAGGTT-3'. GAPDH was used as housekeeping gene, forward $5^{\prime}$-CAAGGCTGTGGGCAAGGT- $3^{\prime}$ and reverse $5^{\prime}$-GGAAGGCCATGCCAGTGA-3'.

2.4. Western Blotting Analysis. 16HBE14o- cells were plated in 6 -well plates and, after $24 \mathrm{~h}$, the growth media were replaced with free-serum and grown overnight. Cells harvested after treatment with inhibitor or AITC were gently washed with ice-cold PBS, and they were placed on ice for $10 \mathrm{~min}$ and suspended in $1 \mathrm{x}$ cell lysis buffer (Invitrogen), and it was supplemented with $2 \mathrm{mM}$ phenylmethanesulfonyl fluoride (PMSF) and a proteinase inhibitor mixture. The protein concentration of the supernatant was measured using BCA reagents (Pierce, Rockford, IL). Proteins were separated by running through 6\% SDS-PAGE gel and transferred to a polyvinylidene difluoride membrane. After being blocked with $5 \%$ nonfatted milk, the membrane was probed by specific antibodies for $1 \mathrm{~h}$ at $37^{\circ} \mathrm{C}$ and $4^{\circ} \mathrm{C}$ overnight and the bands were visualized using the ECL Plus kit according to the manufacturer's instructions. Kodak 1D image analysis software was used to analyse the Western blotting results.

2.5. Flow Cytometry. The impact of JNK pathway on MRP1 activity was examined using a 5-CFDA efflux assay in 16HBE14o- cells with AITC. When 5-CFDA diffuses freely into cells, it is converted to carboxyfluorescein (CF) which is a substrate of MRP1 for efflux. Confluent cells were preloaded with $1 \mu \mathrm{M} 5$-CFDA for $1 \mathrm{~h}$. The cells were incubated with AITC $(40 \mu \mathrm{M})$ for $24 \mathrm{~h}$ after pretreatment with or without the addition of SP600125 $(20 \mu \mathrm{M})$ for $60 \mathrm{~min}$. After incubation, cells were detached and centrifuged at $500 \times \mathrm{g}$ for $5 \mathrm{~min}$, and 


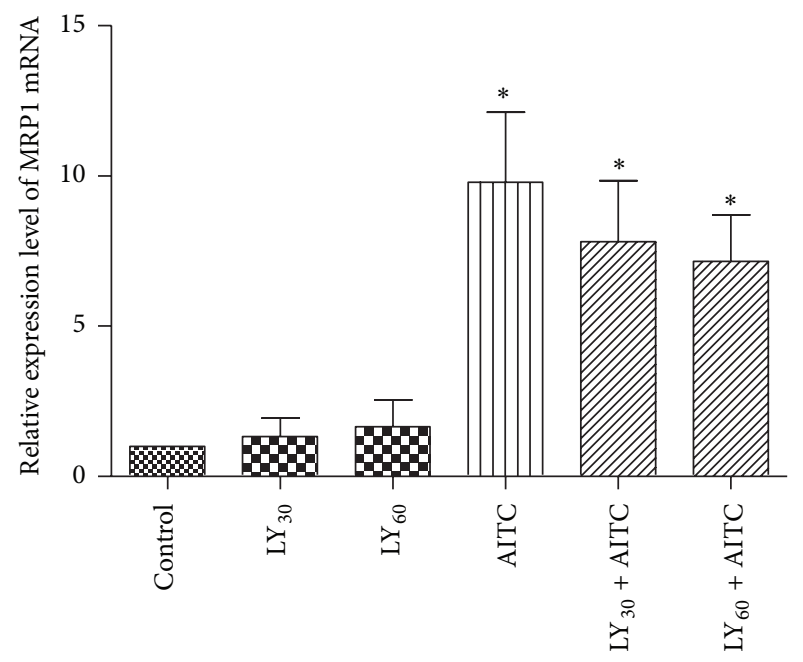

(a)

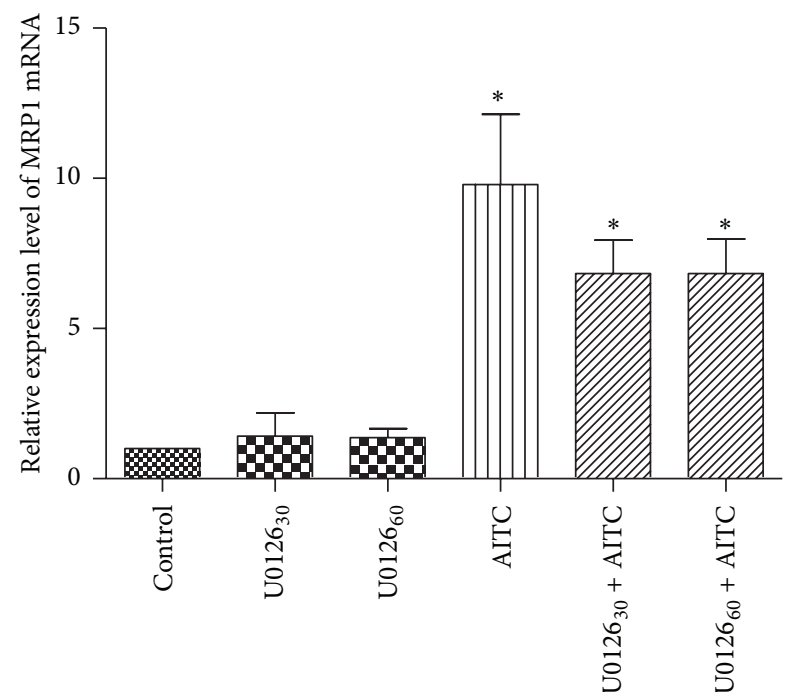

(b)

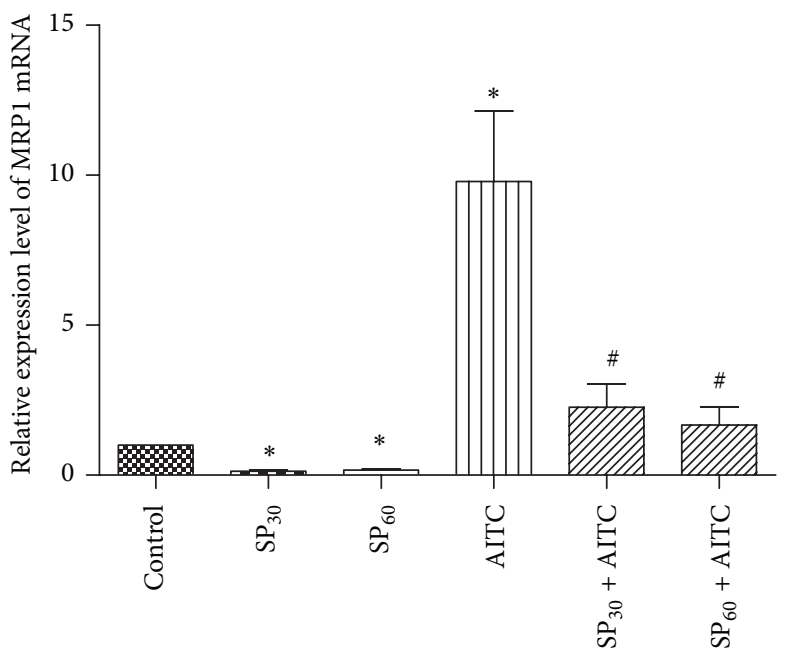

(c)

FIGURE 1: Effect of cell signaling pathway inhibitor on MRP1 mRNA expression in 16HBE14o- cells. (a) Cells were pretreated with or without PI3K/Akt inhibitor LY294002 $(10 \mu \mathrm{M}) 30 \mathrm{~min}$ or $60 \mathrm{~min}$ prior to the exposure to AITC for $24 \mathrm{~h}$, and then the relative expression level of MRP1 was measured by RT-PCR. (b) Cells were pretreated with or without ERK inhibitor U0126 $(20 \mu \mathrm{M}) 30$ min or 60 min prior to the exposure to AITC for $24 \mathrm{~h}$, and then the relative expression level of MRP1 was measured by RT-PCR. (c) Cells were pretreated with or without JNK inhibitor SP600125 $(20 \mu \mathrm{M})$ for $30 \mathrm{~min}$ or $60 \mathrm{~min}$ prior to the exposure to AITC for $24 \mathrm{~h}$, and then the relative expression level of MRP1 was measured by RT-PCR. *Significant difference from control, $P<0.05 .{ }^{\#}$ Significant difference from corresponding AITC control, $P<0.05$.

the pellets were suspended in $1 \mathrm{~mL}$ of ice-cold PBS and immediately placed on ice. Intracellular 5-carboxyfluorescein (5$\mathrm{CF})$ fluorescence intensity was measured using flow cytometry for 30 min using an ABI LSRII flow cytometer (Applied Biosystems). We measured 10,000 events per sample (living cells). Samples were excited at $488 \mathrm{~nm}$ using an argon laser, and the emission fluorescence was detected at $530 \mathrm{~nm}$. The Winlist 5.1 program (Verity Software House Inc., Topsham, ME, USA) was used to calculate the mean fluorescence intensity (MFI) values.

2.6. Statistical. Analysis Student'st-test and one-way ANOVA were used to calculate significant differences. Differences were considered significant when $P<0.05$. Statistical analyses were performed with SPSS 17.0 (SPSS Inc., Chicago, IL).

\section{Results}

3.1. AITC Induced MRP1 Gene Expression via JNK but Not ERK or PI3K/Akt Pathways. To reveal the mechanism of MRP1 mRNA expression induced by AITC, 16HBE14o- cells were pretreated with JNK, ERK, and PI3K/Akt inhibitors for $30 \mathrm{~min}$ or $60 \mathrm{~min}$ prior to the exposure to AITC for $24 \mathrm{~h}$. As shown in Figures 1(a) and 1(b), MRP1 mRNA expression in 16HBE140- cells was not obviously affected by application of ERK inhibitor U0126 $(20 \mu \mathrm{M})$ or PI3K/Akt inhibitor LY294002 $(10 \mu \mathrm{M})$ for $30 \mathrm{~min}$ or $60 \mathrm{~min}$. However, 
TABLE 1: Effect of cell signaling pathway inhibitor on MRP1 $m R N A$ expression.

\begin{tabular}{|c|c|c|c|c|c|}
\hline & GAPDH & MRP1 & $\Delta \mathrm{Ct}$ & $\Delta \Delta \mathrm{Ct}$ & $\mathrm{RQ}=2^{-\Delta \Delta \mathrm{Ct}}$ \\
\hline Control & $15.12 \pm 0.52$ & $19.72 \pm 0.50$ & $4.60 \pm 0.41$ & 0 & 1 \\
\hline AITC & $14.96 \pm 0.67$ & $16.30 \pm 0.58$ & $1.34 \pm 0.17$ & $-3.27 \pm 0.34$ & $9.79 \pm 2.34^{*}$ \\
\hline $\mathrm{LY}_{30}$ & $15.23 \pm 0.45$ & $19.53 \pm 0.94$ & $4.31 \pm 1.03$ & $-0.29 \pm 0.72$ & $1.33 \pm 0.62$ \\
\hline $\mathrm{LY}_{30}+\mathrm{AITC}$ & $14.85 \pm 0.66$ & $16.54 \pm 0.49$ & $1.68 \pm 0.20$ & $-2.92 \pm 0.38$ & $7.73 \pm 2.13^{*}$ \\
\hline $\mathrm{LY}_{60}$ & $14.97 \pm 0.71$ & $19.00 \pm 1.36$ & $4.02 \pm 1.16$ & $-0.58 \pm 0.87$ & $1.67 \pm 0.90$ \\
\hline $\mathrm{LY}_{60}+\mathrm{AITC}$ & $15.36 \pm 0.76$ & $17.51 \pm 0.96$ & $1.79 \pm 0.26$ & $-2.81 \pm 0.32$ & $7.16 \pm 1.55^{*}$ \\
\hline $\mathrm{U}_{30}$ & $14.79 \pm 0.24$ & $19.03 \pm 1.39$ & $4.24 \pm 1.15$ & $-0.36 \pm 0.74$ & $1.41 \pm 0.78$ \\
\hline $\mathrm{U}_{30}+\mathrm{AITC}$ & $14.83 \pm 0.54$ & $16.67 \pm 1.05$ & $1.84 \pm 0.59$ & $-2.76 \pm 0.25$ & $6.83 \pm 1.11^{*}$ \\
\hline $\mathrm{U}_{60}$ & $14.74 \pm 0.70$ & $18.92 \pm 1.02$ & $4.18 \pm 0.64$ & $-0.42 \pm 0.35$ & $1.37 \pm 0.31$ \\
\hline $\mathrm{U}_{60}+\mathrm{AITC}$ & $14.95 \pm 0.62$ & $16.79 \pm 0.65$ & $1.87 \pm 0.37$ & $-2.76 \pm 0.23$ & $6.84 \pm 1.15^{*}$ \\
\hline $\mathrm{SP}_{30}$ & $15.58 \pm 0.18$ & $23.05 \pm 0.70$ & $7.47 \pm 0.79$ & $2.87 \pm 0.42$ & $0.14 \pm 0.04^{*}$ \\
\hline $\mathrm{SP}_{30}+\mathrm{AITC}$ & $15.45 \pm 0.66$ & $19.13 \pm 0.58$ & $3.68 \pm 0.51$ & $-0.92 \pm 0.17$ & $2.26 \pm 0.78^{\#}$ \\
\hline $\mathrm{SP}_{60}$ & $15.56 \pm 0.45$ & $22.71 \pm 0.68$ & $7.15 \pm 0.30$ & $2.54 \pm 0.35$ & $0.18 \pm 0.04^{*}$ \\
\hline $\mathrm{SP}_{60}+\mathrm{AITC}$ & $15.12 \pm 0.61$ & $19.04 \pm 0.39$ & $3.92 \pm 0.81$ & $-0.68 \pm 0.55$ & $1.68 \pm 0.60^{\#}$ \\
\hline
\end{tabular}

Data were normalized against control and presented mean \pm S.D. Experiments were performed in triplicate.

${ }^{*}$ Significant difference from control, $P<0.05$.

${ }^{\#}$ Significant difference from corresponding AITC control, $P<0.05$.

incubation of bronchial epithelial cells with JNK inhibitor SP600125 $(20 \mu \mathrm{M})$ significantly attenuated MRP1 mRNA level (Figure 1(c)). To further examine whether these pathways are involved in AITC-induced MRP1 expression, we observed the effect of specific inhibitors of PI3K/Akt, ERK, and JNK on AITC-induced MRP1 mRNA expression. AITC significantly increased MRP1 mRNA expression (Figures 1(a)1(c)); Table 1. AITC-induced expression was significantly depressed by SP600125 $(20 \mu \mathrm{M})$ (Figure 1(c)). In contrast, $\mathrm{U} 0126(20 \mu \mathrm{M})$ and inhibitor LY294002 $(10 \mu \mathrm{M})$ produced little effect on AITC-induced MRP1 mRNA expression (Figures 1(a) and 1(b)). Together, these results indicated that JNK but not ERK or PI3K/Akt pathway contributes to AITC-induced MRP1 mRNA expression.

3.2. AITC Induced the Protein Expression of MRP1 via JNK Signaling Pathway. We then examined whether AITC affected MRP1 activity due to the upregulated MRP1 mRNA expression following JNK pathway activation. 16HBE14o- cells were pretreated with SP600125 for 60 min before treatment with or without AITC $(40 \mu \mathrm{M})$ for $24 \mathrm{~h}$. The phosphorylation status of JNK was measured using antibody that specifically recognizes the phosphorylated form of JNK. The ratio of phosphorylated JNK to total JNK (p-JNK/t-JNK) was used to evaluate the activity of JNK.

SP600125 alone significantly decreased the expression of MRP1 protein in 16HBE14o- cells (Figure 2(b)). In contrast, AITC $(40 \mu \mathrm{M})$ significantly increased MRP1 protein and the increase was remarkably inhibited by pretreatment with SP600125 (Figure 2(b)). In the meantime, AITC (40 $\mu \mathrm{M})$, but not the vehicle control (DMSO) or JNK inhibitor, significantly increased JNK phosphorylation and the ratio of $\mathrm{p}$ JNK/t-JNK (Figure 2(c)). Pretreatment of SP600125 (20 $\mu \mathrm{M})$ inhibited the upregulation of $\mathrm{p}$-JNK/t-JNK ratio by AITC. These results suggest that activated JNK signaling pathway contributes to the upregulation of MRP1 protein by AITC, supporting the functional role of increased MRP1 mRNA expression.

3.3. Effect of JNK Inhibitor on AITC-Induced MRP1 Function in 16HBE14o-Cells. To determine the role of JNK signaling pathway on AITC-induced MRP1 function, 16HBE140- cells were treated with JNK inhibitor SP600125 (20 $\mu \mathrm{M})$. 5-CFDA was always used as a model MRP1 substrate to evaluate the function of MRP1 [23]. Figure 3(a) depicts the intracellular fluorescence of CF which was measured with flow cell cytometry when cells were incubated with AITC $(40 \mu \mathrm{M})$ with or without pretreatment of SP600125 $(20 \mu \mathrm{M})$. Intracellular fluorescence was significantly increased when cells were loaded with 5-CFDA (Figure 3(a)), suggesting that 5-CFDA were effectively permeated to cells. AITC treatment significantly decreased intracellular CF fluorescence compared to 5-CFDA treatment alone (Figure 3(b)), suggesting a higher function of MRP1. However, the AITC-induced decrease of intracellular CF fluorescence was significantly antagonized by pretreatment of SP600125 $(20 \mu \mathrm{M})$ (Figure 3(b)), indicating that AITC-induced increase of MRP1 function was inhibited by SP600125. These results confirmed that the pharmacological blockade of JNK signal pathway plays an important role in the regulation of AITC-induced MRP1 function.

\section{Discussion}

ABC transporters (i.e., MRP1,P-gp), as an ATP-dependent pump, act as a gatekeeper against numerous xenobiotics, secretion of toxic compounds, apoptosis, and the immune response $[24,25]$. MRP1 is also associated with lung function and inflammatory markers in COPD patients [26]. The clinically used pulmonary drugs such as budesonide, formoterol, and ipratropium bromide are very likely to affect MRP1 activity, besides their positive effects on respiratory symptoms [2]. Some endogenous substances such as the proinflammatory 


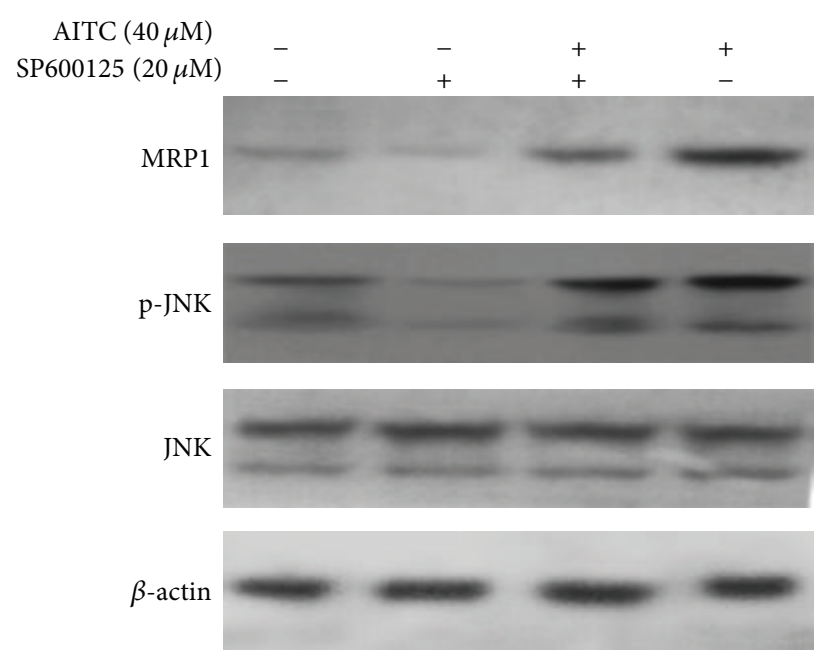

(a)

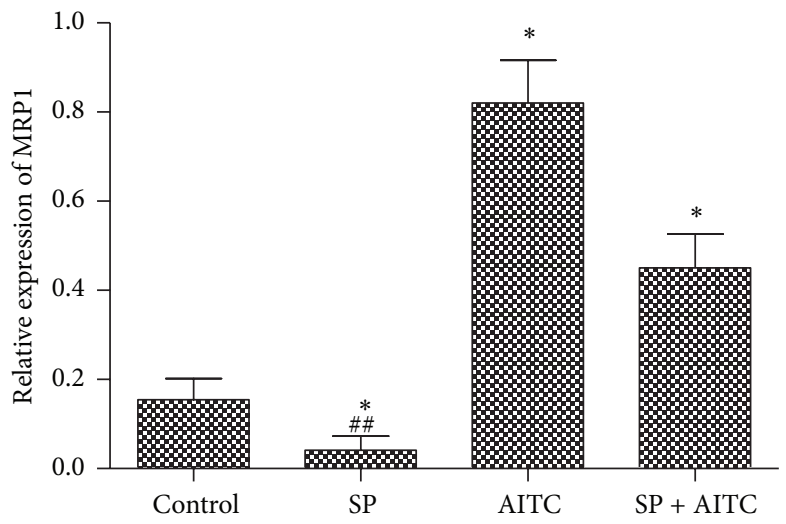

(b)

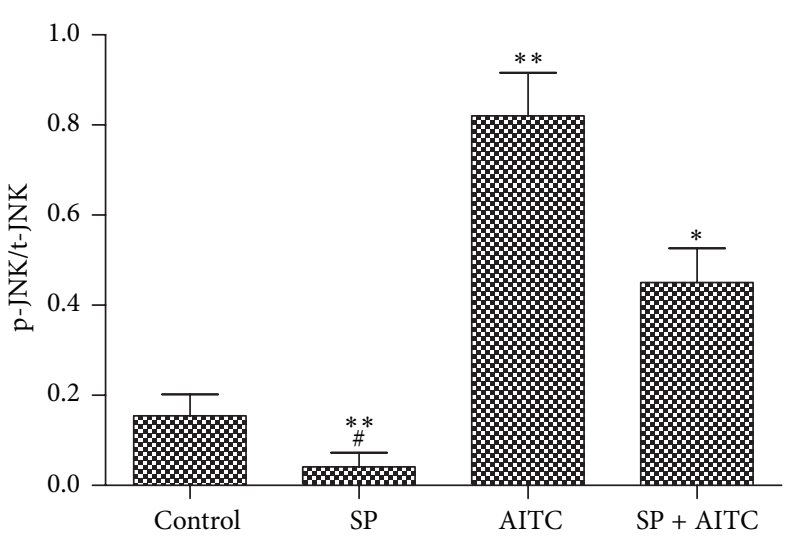

(c)

FIGURE 2: JNK signaling pathway is required for the expression of MRP1 induced by AITC. (a) 16HBE14o- cells were pretreated with SP600125 for $60 \mathrm{~min}$ before presence or absence of $40 \mu \mathrm{M}$ AITC for $24 \mathrm{~h}$, and then the expression of MRP1 protein was measured by Western blot. Densitometric analysis of data from 16HBE14o- cells shows the effects of AITC and/or JNK on MRP1 levels (b) and relative expression of pJNK/t-JNK (c). Data was normalized against control and presented as the mean \pm S.D. Experiments were performed in triplicate. ${ }^{*}$ Significant difference from control, $P<0.05 .{ }^{* *}$ Significant difference from control, $P<0.01$. ${ }^{\#}$ Significant difference from corresponding controls $(\mathrm{SP}+$ AITC), $P<0.05 .{ }^{\#}$ Significant difference from corresponding controls (SP + AITC), $P<0.01$.

leukotriene $\mathrm{C}(4)$ and antioxidant glutathione are known substrates for MRP1 and have a close relationship with COPD [27-29]. Therefore, to better understand the mechanism of COPD, it is important to know how the MRP1 expression is regulated. In vitro and in vivo studies have shown that AITC upregulated the expression and function of MRP1 [22]. Our present findings, for the first time, indicate that JNK MAP kinase pathway contributes to AITC-induced MRP1 expression in human bronchial epithelial cell.

Multiple mechanisms were reported to be involved in the cellular response to AITC. Previous studies indicated that PEITC and/or its conjugates are MRP1 substrates, suggesting that binding interactions with the unmodified molecules was involved in MRP1 inhibition [30]. However, the underlying mechanisms for the function and expression of MRP1 is still unclear. Recently, accumulating evidences suggest that the activation of ERK, JNK, or PI3K/Akt signaling pathway may play an important role in chemoresistance of several cancers cells [7, 12, 31]. U0126 (3-20 $\mu \mathrm{M})$, LY294002 (5-10 $\mu \mathrm{M})$, and SP600125 $(10-20 \mu \mathrm{M})$ were found to selectively inhibited ERK, PI3K/Akt, and JNK pathway, respectively $[9,32,33]$. In this study, we thus used the selective inhibitors to examine the effect of the activation of ERK, JNK, and PI3K/Akt pathways on AITC-induced upregulation of MRP1 expression. Our results indicated that JNK but not ERK or PI3K/Akt pathway plays an important role in MRP1 mRNA expression. In addition, AITC increased MRP1 mRNA expression in 16HBE14ocells. Inhibition of JNK signaling pathway by selective JNK pathway inhibitor SP600125 abolished AITC-induced upregulation of MRP1 mRNA level. However, ERK and PI3K/Akt pathways produced no obvious effect. We also tested the effect of JNK pathway on phosphorylated JNK based on the result that JNK pathway regulated AITC-induced MRP1 mRNA. In contrast to the inhibition of SP600125 on JNK pathway activation, AITC reversed SP600125-inhibition on the activity of JNK signaling which is important in regulating cell apoptosis 

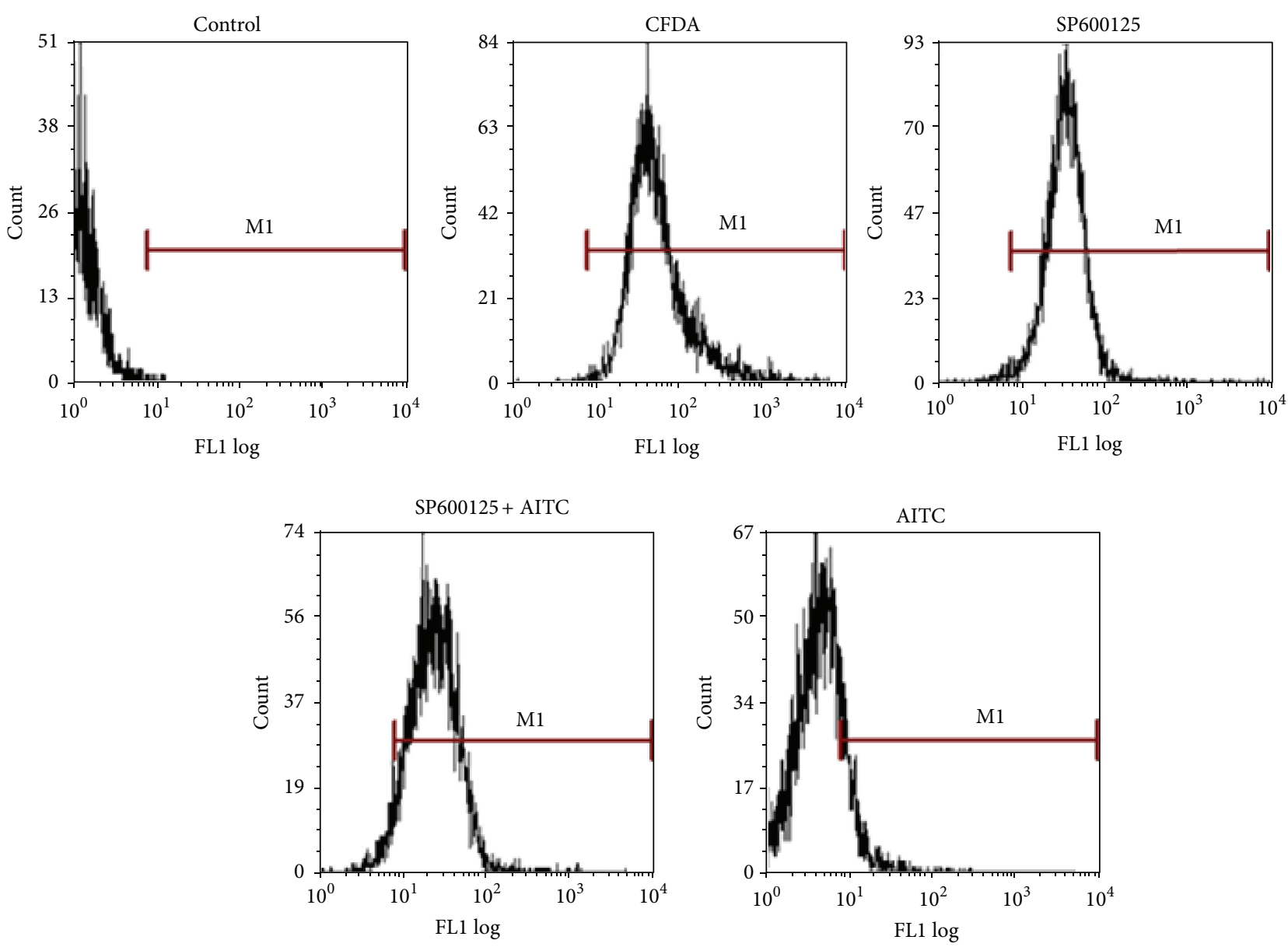

(a)

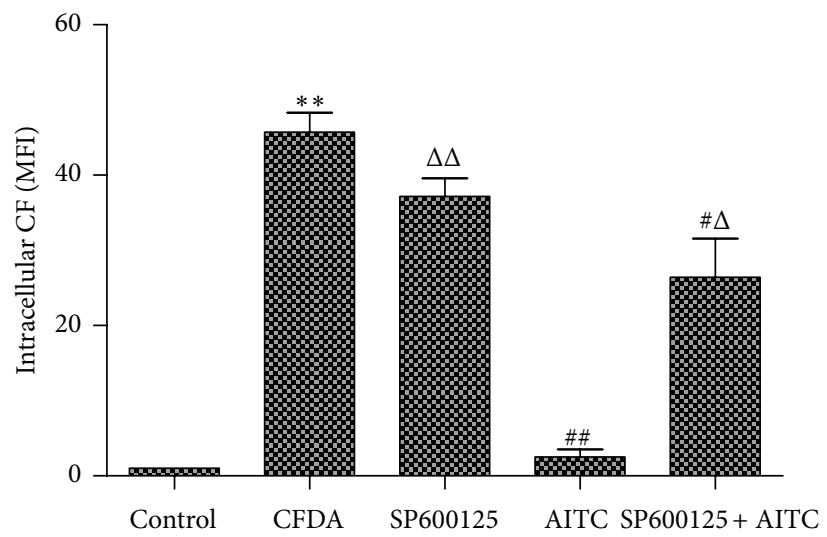

(b)

FIGURE 3: Effect of JNK cell signaling pathway inhibitor on MRP1 function in 16HBE14o- cells. (a) Cells were pretreated with or without the addition of SP600125 $(20 \mu \mathrm{M})$ for 60 min and then incubated with AITC $(40 \mu \mathrm{M})$ for $24 \mathrm{~h}$ and the fluorescence of the CF retained within the cells was evaluated by flow cytometry. (b) Intracellular CF (MFI) retention as a measure of MRP1 function in 16HBE14o- cells. ${ }^{* *}$ Significant difference from control, $P<0.01 .{ }^{\#}$ Significant difference from CFDA, $P<0.05 .{ }^{\# \#}$ Significant difference from CFDA, $P<0.01 .{ }^{\Delta}$ Significant difference from AITC alone, $P<0.05 .{ }^{\Delta \Delta}$ Significant difference from AITC alone, $P<0.01$.

and stress responses [34]. As MRP1 is a protective protein for protecting tissues from toxic xenobiotics and endogenous metabolites [2,3], its expression and activity are critical for COPD. AITC decreased CF accumulation by upregulating
MRP1 function in 16HBE14o- cells. However, AITC-induced decrease of CF was reversed by SP600125. Based on the results, we could reasonably make the conclusion that AITC upregulation of MRP1 is JNK pathway-dependent. JNK 
signaling pathway was reported to be required for MRP1 expression by another compound which has been reported in other cell systems [12]. Tang et al. reported that phenethyl isothiocyanate, one of the isothiocyanates, was found to downregulated multidrugs resistance 1 and MRP1 expression through blocking Akt and activating JNK pathway in T24/ Adriamycin cells [35]. Therefore, our present study for the first time confirmed that AITC increase MRP1 expression in human bronchial epithelial cells through activation of JNK pathway. Previous study indicated that PI3K/Akt signaling pathway participated in regulating MRP1 expression by isothiocyanates [20]. However, our results showed no obvious effect of AITC on MRP1 expression in 16HBE14o- cells.

Smoking is not the only part factor of smokers developing COPD, but it is the main principal risk factor [24]. However, little is known about in vivo detoxification and elimination processes of noxious substances after cigarette smoke is absorbed in lung. During the development of COPD, cigarette smoke extract was reported to affect the protective activity of MRP1 on lung tissue [24]. Additional studies reported that JNK expression in lung parenchyma was increased after tobacco smoke exposure for 5 days in rats and $4-12$ weeks in guinea pigs $[36,37]$. In contrast, upregulation of phosphorylated keratin type 2 cytoskeletal 8 (K8) and keratin type 1 cytoskeletal 18 (K18) is to moderate JNK signaling in lung tissue of rats after a short time of tobacco smoke expo -sure [38]. Taking all into consideration, what happens to the activity of JNK and the downstream events in response to tobacco smoke and/or pretreatment with AITC is not clear. Therefore, it is important to study the regulation mechanism about AITC-regulated MRP1 in stimulation with cigarette smoke extract.

In summary, our study indicated that AITC enhanced the expression of MRP1 at both mRNA level and protein level via JNK signaling pathway but not PI3K/Akt or ERK pathway in 16HBE14o- cells. Understanding the mechanisms of MRP1 regulated by AITC may help us to produce a new therapeutic approach for the reversal and prevention of COPD.

\section{Disclosure}

Shujun Wang and Shanshan Wang were joint first authors.

\section{Conflict of Interests}

The authors declare that there is no conflict of interests regarding the publication of this paper.

\section{Authors' Contribution}

Shujun Wang and Shanshan Wang contributed equally to this work.

\section{Acknowledgments}

This study was financially supported by the National Natural Science Foundation of China (nos. 81473536, 81001592, and
81373598), the Key Project of the Chinese Ministry of Education (no. 210101), and the Key Project of the Anhui University Provincial Natural Science Research Foundation (no. KJ2010A210).

\section{References}

[1] N. Angelis, K. Porpodis, P. Zarogoulidis et al., "Airway inflammation in chronic obstructive pulmonary disease," Journal of Thoracic Disease, vol. 6, supplement 1, pp. S167-S172, 2014.

[2] M. van der Deen, S. Homan, H. Timmer-Bosscha et al., "Effect of COPD treatments on MRP1-mediated transport in bronchial epithelial cells," International Journal of COPD, vol. 3, no. 3, pp. 469-475, 2008.

[3] F. J. Sharom, "ABC multidrug transporters: structure, function and role in chemoresistance," Pharmacogenomics, vol. 9, no. 1, pp. 105-127, 2008.

[4] M. van der Deen, H. Marks, B. W. M. Willemse et al., "Diminished expression of multidrug resistance-associated protein 1 (MRP1) in bronchial epithelium of COPD patients," Virchows Archiv, vol. 449, no. 6, pp. 682-688, 2006.

[5] D.-L. Wang, X. Zhang, X.-H. Tao et al., "Effects of huatan jiangqi capsule on the levels of multi-drug resistance-associated protein 1 in the bronchial epithelial cells of model rats with chronic obstructive pulmonary disease," Zhong guo Zhong Xi Yi Jie He Za Zhi, vol. 32, no. 7, pp. 955-959, 2012.

[6] S. P. C. Cole, "Targeting multidrug resistance protein 1 (MRP1, ABCC1): past, present, and future," Annual Review of Pharmacology and Toxicology, vol. 54, pp. 95-117, 2014.

[7] S. Lin, K. Hoffmann, Z. Xiao et al., "MEK inhibition induced downregulation of MRP1 and MRP3 expression in experimental hepatocellular carcinoma," Cancer Cell International, vol. 13, no. 1, article 3, 2013.

[8] F. Yan, X.-M. Wang, Z.-C. Liu, C. Pan, S.-B. Yuan, and Q.-M. $\mathrm{Ma}$, "JNK1, JNK2, and JNK3 are involved in P-glycoproteinmediated multidrug resistance of hepatocellular carcinoma cells," Hepatobiliary and Pancreatic Diseases International, vol. 9, no. 3, pp. 287-295, 2010.

[9] H. Ma, L. Cheng, K. Hao et al., "Reversal effect of ST6GAL 1 on multidrug resistance in human leukemia by regulating the PI3K/Akt pathway and the expression of P-gp and MRP1," PLoS ONE, vol. 9, no. 1, Article ID e85113, 2014.

[10] G. Mordret, "MAP kinase kinase: a node connecting multiple pathways," Biology of the Cell, vol. 79, no. 3, pp. 193-207, 1993.

[11] G. L'Allemain, "Deciphering the MAP kinase pathway," Progress in Growth Factor Resarch, vol. 5, no. 3, pp. 291-334, 1994.

[12] P. T. Ronaldson, T. Ashraf, and R. Bendayan, "Regulation of multidrug resistance protein 1 by tumor necrosis factor $\alpha$ in cultured glial cells: involvement of nuclear factor- $\kappa \mathrm{B}$ and c-Jun Nterminal kinase signaling pathways," Molecular Pharmacology, vol. 77, no. 4, pp. 644-659, 2010.

[13] A. Bellacosa, C. C. Kumar, A. Di Cristofano, and J. R. Testa, "Activation of AKT kinases in cancer: implications for therapeutic targeting," Advances in Cancer Research, vol. 94, no. 1, pp. 29-86, 2005.

[14] M. G. García, L. D. Alaniz, R. I. Cordo Russo, E. Alvarez, and S. E. Hajos, "PI3K/Akt inhibition modulates multidrug resistance and activates NF- $\kappa \mathrm{B}$ in murine lymphoma cell lines," Leukemia Research, vol. 33, no. 2, pp. 288-296, 2009.

[15] J. Li, X. Wu, J. Gong et al., "Vascular endothelial growth factor induces multidrug resistance-associated protein 1 overexpression through phosphatidylinositol-3-kinase/protein kinase $\mathrm{B}$ 
signaling pathway and transcription factor specificity protein 1 in BGC823 cell line," Acta Biochimica et Biophysica Sinica, vol. 45, no. 8, pp. 656-663, 2013.

[16] J. Geisel, J. Brück, I. Glocova et al., "Sulforaphane protects from T cell-mediated autoimmune disease by inhibition of IL-23 and IL-12 in dendritic cells," The Journal of Immunology, vol. 192, no. 8, pp. 3530-3539, 2014.

[17] F. Zanichelli, S. Capasso, G. Di Bernardo et al., "Low concentrations of isothiocyanates protect mesenchymal stem cells from oxidative injuries, while high concentrations exacerbate DNA damage," Apoptosis, vol. 17, no. 9, pp. 964-974, 2012.

[18] L. Mi and F.-L. Chung, "Binding to protein by isothiocyanates: a potential mechanism for apoptosis induction in human nonsmall lung cancer cells," Nutrition and Cancer, vol. 60, supplement 1, pp. 12-20, 2008.

[19] F. Yan, Y. Jiang, Y.-M. Li, X. Zhen, J. Cen, and W.-R. Fang, "Reversal of P-glycoprotein and multidrug resistance-associated protein 1 mediated multidrug resistance in cancer cells by HZ08 isomers, tetrataisohydroquinolin derivatives," Biological \& Pharmaceutical Bulletin, vol. 31, no. 6, pp. 1258-1264, 2008.

[20] T. Tang, X. Song, Y.-F. Liu, and W.-Y. Wang, "PEITC reverse multi-drug resistance of human gastric cancer SGC7901/DDP cell line," Cell Biology International, vol. 38, no. 4, pp. 502-510, 2014.

[21] Y. Cao, Investigate the mechanism of allyl isothiocyanate in treatment of COPD based on its pharmacokinetics and the effect on the function of MRP1 [M.S. dissertation], Anhui University of Chinese Medicine, 2013.

[22] D.-L. Wang, C.-Y. Wang, Y. Cao et al., "Allyl isothiocyanate increases MRP1 function and expression in a human bronchial epithelial cell Line," Oxidative Medicine and Cellular Longevity, vol. 2014, Article ID 547379, 7 pages, 2014.

[23] B. Laupeze, L. Amiot, A. Courtois et al., "Use of the anionic dye carboxy-2 $2^{\prime}, 7^{\prime}$-dichlorofluorescein for sensitive flow cytometric detection of multidrug resistance-associated protein activity.", International Journal of Oncology, vol. 15, no. 3, pp. 571-576, 1999.

[24] M. van der Deen, E. G. E. de Vries, H. Visserman et al., "Cigarette smoke extract affects functional activity of MRP1 in bronchial epithelial cells," Journal of Biochemical and Molecular Toxicology, vol. 21, no. 5, pp. 243-251, 2007.

[25] M. Milojkovic, N. Milacic, J. Radovic et al., "MDR1 gene polymorphisms and P-glycoprotein expression in respiratory diseases," Biomedical Papers of the Medical. Faculty of the University. Palacky Olomouc Czechoslovakia, 2014.

[26] S. E. Budulac, D. S. Postma, P. S. Hiemstra et al., "Multidrug resistance-associated protein 1 and lung function decline with or without long-term corticosteroids treatment in COPD," European Journal of Pharmacology, vol. 696, no. 1-3, pp. 136142, 2012.

[27] S.-F. Zhou, L.-L. Wang, Y. M. Di et al., "Substrates and inhibitors of human multidrug resistance associated proteins and the implications in drug development," Current Medicinal Chemistry, vol. 15, no. 20, pp. 1981-2039, 2008.

[28] L. Manciu, X.-B. Chang, F. Buyse et al., "Intermediate structural states involved in MRP1-mediated drug transport. Role of glutathione," The Journal of Biological Chemistry, vol. 278, no. 5, pp. 3347-3356, 2003.

[29] R. G. Deeley and S. P. C. Cole, "Substrate recognition and transport by multidrug resistance protein 1 (ABCC1)," FEBS Letters, vol. 580, no. 4, pp. 1103-1111, 2006.
[30] K. Hu and M. E. Morris, "Effects of benzyl-, phenethyl-, and alpha-naphthyl isothiocyanates on P-glycoprotein- and MRP1mediated transport," Journal of Pharmaceutical Sciences, vol. 93, no. 7, pp. 1901-1911, 2004.

[31] L. Cheng, S. Luo, C. Jin, H. Ma, H. Zhou, and L. Jia, "FUT family mediates the multidrug resistance of human hepatocellular carcinoma via the PI3K/Akt signaling pathway," Cell Death \& Disease, vol. 4, no. 11, article e923, 2013.

[32] Z. Wu, H. Uchi, S. Morino-Koga et al., "Z-Ligustilide inhibits benzo(a)pyrene-induced CYP1A1 upregulation in cultured human keratinocytes via ROS-dependent Nrf2 activation," Experimental Dermatology, vol. 23, no. 4, pp. 260-265, 2014.

[33] R. Garcia, R. A. Franklin, and J. A. McCubrey, "EGF induces cell motility and multi-drug resistance gene expression in breast cancer cells," Cell Cycle, vol. 5, no. 23, pp. 2820-2826, 2006.

[34] A. Munshi and R. Ramesh, "Mitogen-activated protein kinases and their role in radiation response," Genes \& Cancer, vol. 4, no. 9-10, pp. 401-408, 2013.

[35] K. Tang, Y. Lin, and L.-M. Li, “The role of phenethyl isothiocyanate on bladder cancer $\mathrm{ADM}$ resistance reversal and its molecular mechanism," The Anatomical Record, vol. 296, no. 6, pp. 899-906, 2013.

[36] C. A. Carter and M. Misra, "Effects of short-term cigarette smoke exposure on fischer 344 rats and on selected lung proteins," Toxicologic Pathology, vol. 38, no. 3, pp. 402-415, 2010.

[37] P. Golovatch, B. A. Mercer, V. Lematre, A. Wallace, R. F. Foronjy, and J. D'Armiento, "Role for cathepsin $\mathrm{K}$ in emphysema in smoke-exposed guinea pigs," Experimental Lung Research, vol. 35, no. 8, pp. 631-645, 2009.

[38] K. Niimori-Kita, K. Ogino, S. Mikami et al., "Identification of nuclear phosphoproteins as novel tobacco markers in mouse lung tissue following short-term exposure to tobacco smoke," FEBS Open Bio, vol. 4, pp. 746-754, 2014. 


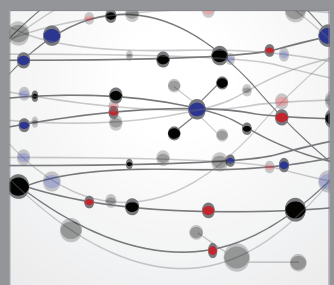

The Scientific World Journal
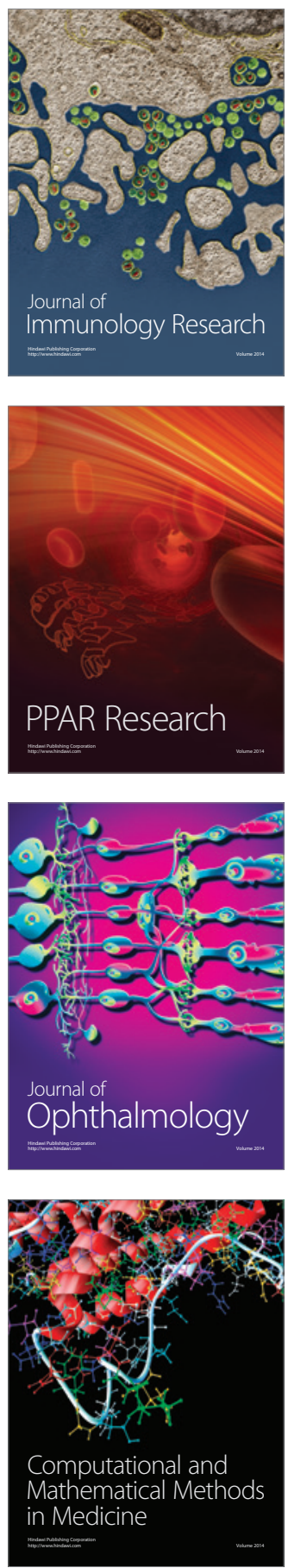

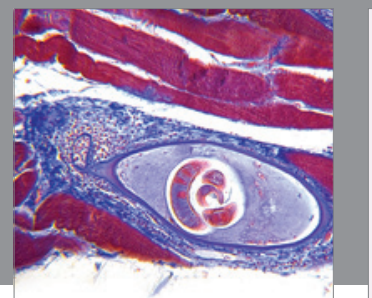

Gastroenterology

Research and Practice
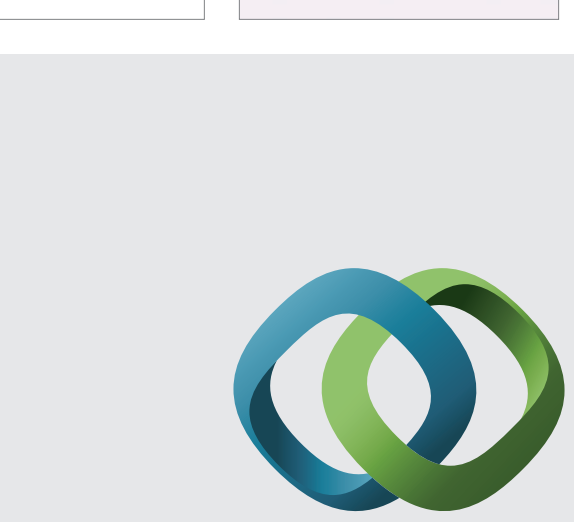

\section{Hindawi}

Submit your manuscripts at

http://www.hindawi.com
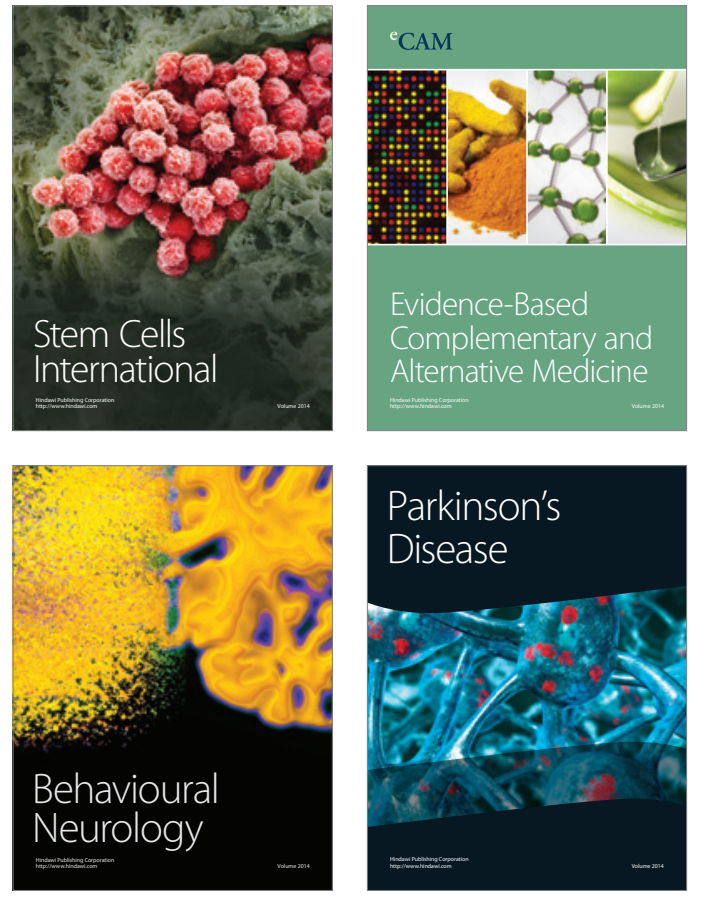
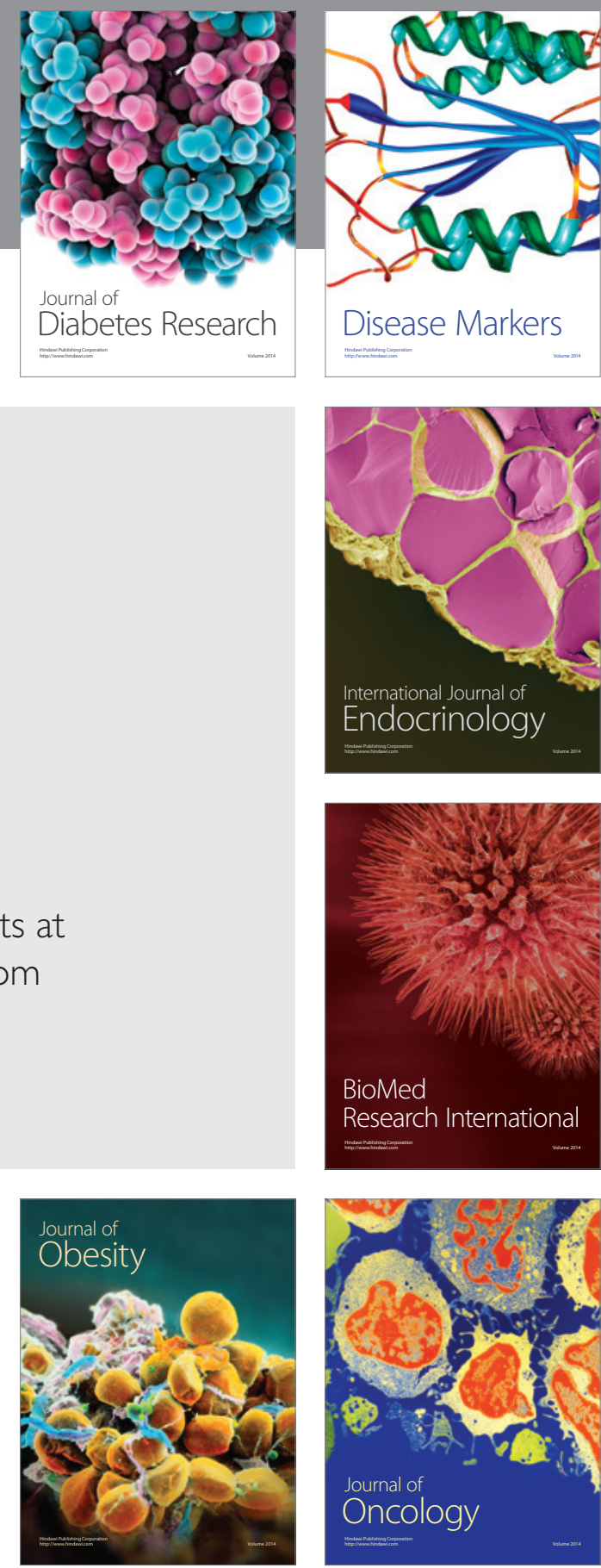

Disease Markers
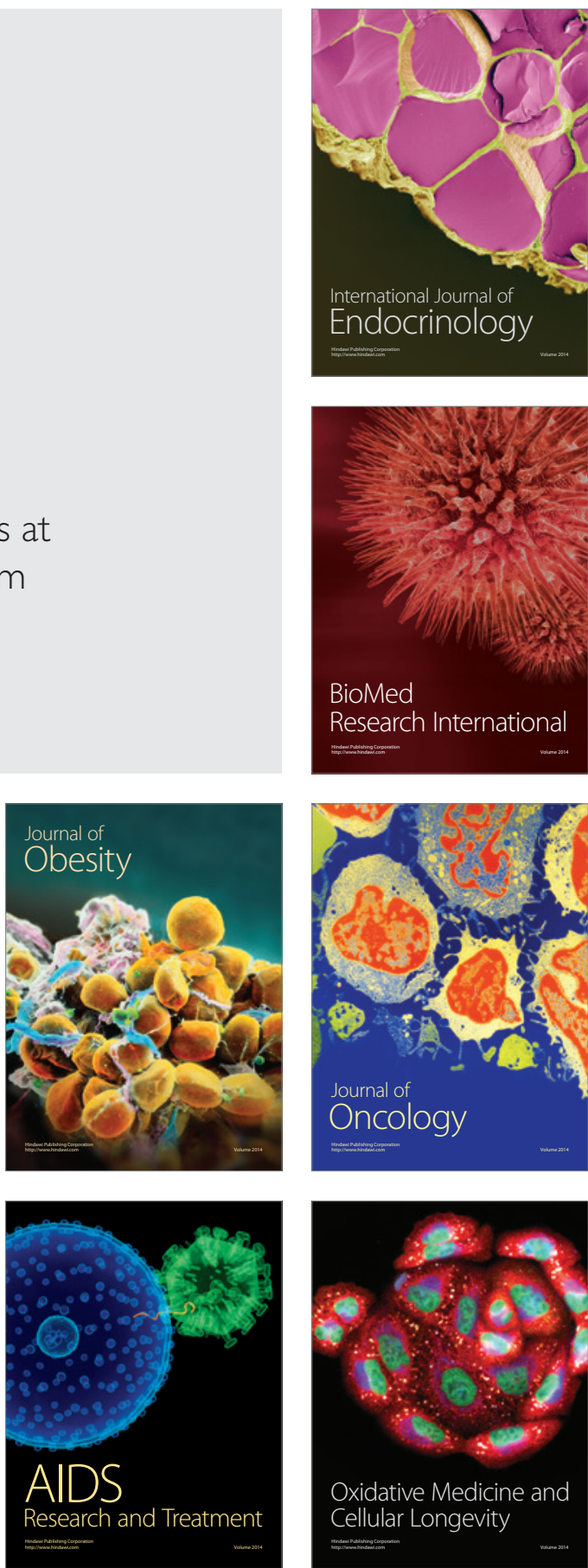